\title{
Controlled entanglement of two field modes in a Cavity Quantum Electrodynamics experiment
}

\author{
A. Rauschenbeutel, P. Bertet, S. Osnaghi, G. Nogues, M. Brune, J.M. Raimond, S. \\ Haroche \\ Laboratoire Kastler Brossel *, \\ Département de Physique de l'Ecole Normale Supérieure, \\ 24 rue Lhomond, F-75231 Paris Cedex 05, France
}

(October 23, 2018)

\begin{abstract}
Using a single circular Rydberg atom, we have prepared two modes of a superconducting cavity in a maximally entangled state. The two modes share a single photon. This entanglement is revealed by a second atom probing, after a delay, the correlations between the two modes. This experiment opens interesting perspectives for quantum information manipulation and fundamental tests of quantum theory.
\end{abstract}

PACS numbers: 03.65.-w, 03.67.-a, 42.50.-p

Typeset using REVTEX

*Unité mixte de recherche, Ecole normale supérieure, Université Pierre et Marie Curie et CNRS (UMR8552) 
The preparation of complex quantum entangled states in well-controlled conditions is the subject of an intense experimental activity. The manipulation of these states which have non-classical and non-local properties leads to a better understanding of basic quantum phenomena. Complex entangled states, such as the Greenberger, Horne and Zeilinger triplets of particles [1] are used for tests of quantum non-locality [2]. Moreover, the relaxation dynamics of large entangled states sheds light on the decoherence process and on the quantum-classical boundary [3].

Beyond these fundamental aspects, entangled quantum states can be used for information processing. Elements of information may be coded on quantum two-level systems ("qubits") [4]. Quantum entanglement between qubits leads to new functions for information processing or transmission. Non-local correlations between two qubits can be used for quantum key distribution [5] or teleportation [6]. More complex entanglement manipulations could be used for quantum error correction [7] or entanglement purification [8]. The manipulation of controlled entangled states, protected from their environment, is experimentally challenging. Clear-cut entanglement between individual systems has been achieved so far only in quantum optics, in photon down-conversion processes [2], with trapped ions [9] or in cavity quantum electrodynamics [10].

In the latter case, the entanglement results from the interaction of a two-level atom with a cavity field mode. With circular Rydberg atoms and superconducting cavities, the coherent atom-field coupling overwhelms dissipation [11]. The basic interaction process is the vacuum Rabi oscillation [12]. An atom, entering the empty cavity in the upper state of the transition resonant with the mode, periodically emits and absorbs a photon. Interrupting the coherent interaction at selected times, one can generate atom-cavity entanglement [13], exchange atom and cavity states [14] or realize a two-qubit quantum logic gate [15], basic unit of quantum information processing systems. These functions can be arranged to realize a quantum non-demolition measurement of a single photon [16] or to generate a three-particle entangled state [10].

Up to now, these experiments have involved a single cavity mode interacting with one, 
two or three atoms crossing it successively. We present here an important step towards the realization of more complex entanglement in cavity QED, by involving in the experiment two independent cavity modes. We have shown that a single atom can be used to entangle the two modes, a second atom being employed later to reveal this entanglement. This multi-mode entanglement opens new perspectives for quantum information processing and non-locality tests.

The principle of the experiment is sketched on figure 1(a). Circular Rydberg atoms cross, one at a time, the superconducting cavity $C$ which sustains two non-degenerate orthogonally polarized modes $M_{a}$ and $M_{b}$, initially in the vacuum state $|0\rangle$. The $M_{a}-M_{b}$ frequency difference is $\delta$ ( $M_{a}$ has the highest frequency). An experimental sequence involves two circular Rydberg atoms $A_{s}$ and $A_{p}$ with two levels $e$ and $g$ (upper and lower states respectively). $A_{s}$ is a source atom which emits a single photon coherently shared between the two cavity modes at different frequencies. Atom $A_{p}$ probes at a later time this single photon state. The final states of both atoms ( $e$ or $g$ ) are analyzed in the state-selective field-ionization detector $D$.

The detuning $\Delta$ between the frequency of the $e \rightarrow g$ transition and mode $M_{a}$ can be tuned through Stark effect. When $\Delta=0(-\delta)$, the atom is in resonance with mode $M_{a}$ $\left(M_{b}\right)$. When the atom is tuned to resonance with one mode, the interaction with the second one has a small effect, provided that $\delta$ is much larger than the vacuum Rabi oscillation frequency $\Omega$. Finally, the detuning $\Delta$ can also be set to a large negative value, freezing the atom-field evolution.

The experiment timing is schematized on figure 1(b). Atom $A_{s}$ enters $C$ in level $e$ at time $t=0$. The initial $A_{s}-M_{a}-M_{b}$ state is $\left|e_{s}, 0_{a}, 0_{b}\right\rangle$. Atom $A_{s}$ interacts first resonantly with $M_{a}$ for a $\pi / 2$ spontaneous emission pulse [12]. Assuming, for sake of simplicity, that the atom-field coupling is constant (we neglect thus the spatial dependence of the cavity mode amplitude), the duration of this Rabi pulse is $\pi / 2 \Omega$. With a proper choice for the atomic dipole phase and taking the energy of state $\left|g_{s}, 1_{a}, 0_{b}\right\rangle$ as the energy origin, the resulting atom-cavity state is, at time $t=\pi / 2 \Omega$ : 


$$
\left|\Psi_{1}\right\rangle=\frac{1}{\sqrt{2}}\left(\left|e_{s}, 0_{a}\right\rangle+\left|g_{s}, 1_{a}\right\rangle\right)\left|0_{b}\right\rangle
$$

Atom $A_{s}$ and mode $M_{a}$ are then in a maximally entangled Einstein-Podolsky-Rosen (EPR) state [17].

The atomic state, and thus its entanglement with $M_{a}$, are then copied onto mode $M_{b}$. Atom $A_{s}$ is tuned to resonance with $M_{b}(\Delta=-\delta)$ and undergoes a $\pi$ spontaneous emission pulse in this mode, performing the transformations $\left|g_{s}, 0_{b}\right\rangle \rightarrow\left|g_{s}, 0_{b}\right\rangle$ and $\left|e_{s}, 0_{b}\right\rangle \rightarrow i \exp (i \delta \pi / \Omega)\left|g_{s}, 1_{b}\right\rangle$. The $i$ phase factor in the latter transformation is due to the fact that the polarization of mode $M_{b}$ is orthogonal to the one of mode $M_{a}$. The other phase factor originates in the $-\hbar \delta$ energy of state $\left|g_{s}, 0_{a}, 1_{b}\right\rangle$. This phase accumulates during the time $\pi / \Omega$ required for a $\pi$ Rabi rotation. Note that we neglect the light shifts produced by $M_{a}$ on the atom during its interaction with $M_{b}$.

Atom $A_{s}$ finally ends up in $g$ and its state can be factorized out. The two modes end up at time $t=3 \pi / 2 \Omega$ in the EPR pair state :

$$
\left|\Psi_{2}(0)\right\rangle=\frac{1}{\sqrt{2}}\left(e^{i \phi}\left|0_{a}, 1_{b}\right\rangle+\left|1_{a}, 0_{b}\right\rangle\right)
$$

where $\phi=\pi / 2+\pi \delta / \Omega$. In terms of quantum information, this is a maximally entangled state of two qubits stored in the two cavity modes, the photon number states $|0\rangle$ and $|1\rangle$ being the qubits logical levels. At a later time $t$, the phase due to the energy difference $\hbar \delta$ between the two modes accumulates futher, for a time interval $t-3 \pi / 2 \Omega$. The two-mode state becomes thus : $\left|\Psi_{2}(t)\right\rangle=\left(i \exp (-i \delta \pi / 2 \Omega) \exp (i \delta t)\left|0_{a}, 1_{b}\right\rangle+\left|1_{a}, 0_{b}\right\rangle\right) / \sqrt{2}$.

The modes entanglement is revealed by sending in $C$ the probe atom $A_{p}$ at time $t=T$. Atom $A_{p}$, initially in $g$, undergoes first a resonant $\pi$ Rabi pulse induced by the photon stored in $M_{a}$ during a time interval $\pi / \Omega$. This pulse copies the state of $M_{a}$ onto $A_{p}$ The resulting $A_{p}-M_{a}-M_{b}$ state is : $\left|\Psi_{3}\right\rangle=\left|0_{a}\right\rangle\left(i \exp (i \delta \pi / 2 \Omega) \exp (i \delta T)\left|g_{p}, 1_{b}\right\rangle-\left|e_{p}, 0_{b}\right\rangle\right) / \sqrt{2}$. Cavity mode $M_{a}$ ends up in $|0\rangle$ and factorizes out.

Atom $A_{p}$ interacts then with $M_{b}$ for a $\pi / 2$ Rabi pulse (duration $\pi / 2 \Omega$ ), performing the transformations $\left|g_{p}, 1_{b}\right\rangle \rightarrow \exp (i \delta \pi / 2 \Omega)\left(i\left|e_{p}, 0_{b}\right\rangle+\left|g_{p}, 1_{b}\right\rangle\right) / \sqrt{2}$ and $\left|e_{p}, 0_{b}\right\rangle \rightarrow$ 
$\exp (i \delta \pi / 2 \Omega)\left(\left|e_{p}, 0_{b}\right\rangle+i\left|g_{p}, 1_{b}\right\rangle\right) / \sqrt{2}$. The final $A_{p}-M_{b}$ state is thus, within a global phase factor :

$$
\begin{aligned}
\left|\Psi_{4}\right\rangle= & \frac{1}{2}\left(i\left|g_{p}, 1_{b}\right\rangle\left(1-e^{i \delta T} e^{i \delta \pi / 2 \Omega}\right)\right. \\
& \left.+\left|e_{p}, 0_{b}\right\rangle\left(1+e^{i \delta T} e^{i \delta \pi / 2 \Omega}\right)\right) .
\end{aligned}
$$

The probability $P_{e}(T)$ for finding $A_{p}$ in $e$ is

$$
P_{e}(T)=(1+\cos (\delta T+\Phi)) / 2
$$

with $\Phi=\pi \delta / 2 \Omega$. It oscillates between zero and one as a function of the time interval $T$. This oscillation reveals the coherent nature of the mode states superposition.

The experimental set-up (figure 1(a)) has essentially been described in [10,12 16]. The rubidium circular levels $e$ and $g$ have principal quantum numbers 51 and 50 respectively $(e \rightarrow g$ transition frequency $51.1 \mathrm{GHz}$ ). The atoms are prepared in zone $B$ by a timeresolved excitation of a velocity-selected $(v=503 \pm 2 \mathrm{~m} / \mathrm{s})$ atomic beam. Atoms $A_{s}$ and $A_{p}$ belong to two circular state samples separated by an adjustable time interval $T$. The position of these two samples at any time is known within $\pm 1 \mathrm{~mm}$, allowing us to control precisely and independently the Rabi pulses. Each atomic sample contains on the average 0.12 atoms. The probability for having two atoms in the same sample is thus low. Data acquisition selects events where a single atom is detected (in $D$ ) in each sample. The atomic path is enclosed in a $1.3 \mathrm{~K}$ cryostat protecting the atoms from the resonant blackbody field. The experimental sequence is repeated at a $600 \mathrm{~s}^{-1}$ rate.

The cavity $C$ is a Fabry Perot resonator made of two spherical niobium mirrors. The two orthogonally polarized $\mathrm{TEM}_{900}$ modes, $M_{a}$ and $M_{b}$, have the same gaussian geometry (waist $w=6 \mathrm{~mm}$ ). The frequency splitting due a slight mirror shape anisotropy, $\delta / 2 \pi=$ $128.3 \pm 0.1 \mathrm{kHz}$, is measured by auxiliary microwave transmission experiments. The photon damping times are $T_{r, a}=1 \mathrm{~ms}$ and $T_{r, b}=0.9 \mathrm{~ms}$ for $M_{a}$ and $M_{b}$ respectively. At thermal equilibrium, the modes contain a small thermal field originating from room-temperature microwave leaks (mean photon numbers 0.8 and 1 for modes $M_{a}$ and $M_{b}$ respectively). Before 
the beginning of the experimental sequence, this thermal field is "erased" down to less than 0.1 photon on average in each mode [16]. It then relaxes back to thermal equilibrium.

A d.c. voltage applied across the mirrors maintains the atomic orbital plane perpendicular to the cavity axis. The atoms are thus coupled in the same way to both modes, with a vacuum Rabi oscillation frequency $\Omega / 2 \pi=47 \mathrm{kHz}$ at cavity center. In this static electric field, the atomic levels undergo a quadratic Stark effect (transition shift $\left.-255 \mathrm{kHz} /(\mathrm{V} / \mathrm{cm})^{2}\right)$. They are in resonance with modes $M_{a}$ and $M_{b}$ in electric fiels equal to $0.26 \mathrm{~V} / \mathrm{cm}$ and 0.76 $\mathrm{V} / \mathrm{cm}$ respectively. The atom-field interaction is interrupted by a $1.1 \mathrm{~V} / \mathrm{cm}$ field, shifting the atomic line $150 \mathrm{kHz}$ below the frequency of $M_{b}(\Delta=-278 \mathrm{kHz})$.

Atom $A_{s}$ enters the cavity at resonance with $M_{a}$. After a $\pi / 2$ spontaneous emission pulse, $A_{s}$ is rapidly (within $1 \mu \mathrm{s}$ ) tuned to resonance with $M_{b}\left(A_{s}\right.$ is then $3 \mathrm{~mm}$ before the cavity axis). During the next $12 \mu \mathrm{s}$ (6 mm path), $A_{s}$ experiences a $\pi$ spontaneous emission pulse in $M_{b}$. The largest Stark field value is then applied, freezing the evolution. Atom $A_{s}$ is detected in the expected level $g$ in $86 \%$ of the cases. The departure from unity originates from detector errors and from the imperfections of the Rabi pulses. Sequences with $A_{s}$ detected in $e$ are discarded. Atom $A_{p}$ is resonant with $M_{a}$ when it enters $C$. The resonance condition is maintained until the atom fullfills the $\pi$ Rabi pulse condition. Atom $A_{p}$ then interacts with $M_{b}$ for a $\pi / 2$ pulse and is finally detuned by a large field.

Figure 2 presents the probability $P_{e}(T)$ for detecting $A_{p}$ in $e$ as a function of $T$. The data sets (a), (b), (c) and (d) correspond to four different windows in the $0 \rightarrow 710 \mu \mathrm{s}$ time interval. The dots are experimental, with error bars reflecting the binomial detection statistics variance. The curves are sine fits. The frequency of these fits is the independently determined $\delta$ value. The four fits have independently adjustable contrasts and offsets. They share a common phase $\Phi$. Since the qualitative model of the experiment presented above does not take into account properly many important effects (spatial dependence of the cavity modes, light shifts...), $\Phi$ does not take the simple $\delta \pi / 2 \Omega$ value predicted by Eq. (丑). We thus adjust $\Phi$ on the experimental data.

We clearly observe the beat note between the two modes sharing a single photon at the 
expected frequency $\delta$. The contrast of the beat decreases with time, due to the cavity modes relaxation towards thermal equilibrium. At very long times, $P_{e}$ reaches a $30 \%$ limit value, due to the absorption of the equilibrium thermal fields in both modes by atom $A_{p}$. Finally, we have checked that $P_{e}(T)$ is not modulated and reaches the same limiting $30 \%$ value when atom $A_{s}$ is not sent.

This experiment shows that two cavity modes can be manipulated and coupled together according to pre-determined schemes. Many variants of this experiment could be implemented, opening new perspectives for quantum information manipulations in cavity QED experiments. For example, a quantum logic gate could be operated with the two modes as qubits. Mode $M_{a}$ and $M_{b}$ would play the role of the control and target qubits respectively. To implement the gate operation, an atom is sent across the cavity and undergoes a $\pi$ Rabi pulse in a one photon $M_{b}$ field. Its state becomes then a copy of the target. The atom is then detuned from the empty $M_{b}$ mode, while remaining non-resonant with $M_{a}$. The atomic coherence thus undergoes a phase shift which depends upon the photon number in $M_{a}$ [18]. In this way, the conditional dynamics of a quantum phase gate can be realized. The modified target state would finally be copied back onto $M_{b}$ by a resonant $\pi$ spontaneous emission pulse. The process can be generalized to couple more qubits. For example, the non-resonant interaction of an atom with both modes acting as joint control qubits leads naturally to three-qubit gates. Combining such gates implying two field modes and up to four atoms makes it possible to implement simple quantum algorithms [19]. Finally, the entangling scheme described in this paper could be applied to modes belonging to two separate cavities, realizing non-local field state entanglement [20]. Such states could be used for teleportation of matter particle states [21].

Acknowledgements This work was supported by the Commission of the European Community and by the Japan Science and Technology Corporation (International Cooperative Research Project, Quantum Entanglement Project). 


\section{REFERENCES}

[1] D.M. Greenberger, M.A. Horne and A. Zeilinger, Am. J. Phys. 58, 1131 (1990).

[2] J.W. Pan et al., Nature, 403, 515 (2000).

[3] W.H. Zurek, Physics Today, 44, 10 p. 36 (1991).

[4] D. P. DiVincenzo, Science 270, 255 (1995); A. Ekert and R. Josza, Rev. Mod. Phys. 68, 3733, (1997).

[5] A. Ekert, Phys. Rev. Lett. 67, 661 (1991).

[6] C.H. Bennett et. al., Phys. Rev. Lett. 70, 1895 (1993).

[7] A. Steane, Phys. Rev. Lett. 77, 793 (1996).

[8] S.J. Van Enk, J.I. Cirac and P. Zoller, Phys. Rev. Lett., 78, 4293 (1997).

[9] C. A. Sackett et al., Nature, 404, 256 (2000).

[10] A. Rauschenbeutel et al., Science, 288, 2024 (2000).

[11] S. Haroche and J.M. Raimond, in Cavity Quantum Electrodynamics, P. Berman ed., Academic Press, p. 123 (1994).

[12] M. Brune et al., Phys. Rev. Lett. 76, 1800 (1996).

[13] E. Hagley et al., Phys. Rev. Lett. 79, 1 (1997).

[14] X. Maître et al., Phys. Rev. Lett. 79, 769 (1997).

[15] A. Rauschenbeutel et al., Phys. Rev. Lett. 83, 5166 (1999).

[16] G. Nogues et al., Nature 400, 239 (1999).

[17] A. Einstein, B. Podolosky and N. Rosen, Phys. Rev. 47, 777 (1935).

[18] M. Brune et al., Phys. Rev. Lett. 65, 976 (1990). 
[19] F. Yamaguchi, Private communication (2000).

[20] P. Meystre, in Progress in Optics XXX, edited by E. Wolf (Elsevier Science, New York, 1992).

[21] L. Davidovich et al., Phys. Rev. A50, R895 (1994). 


\section{FIGURES}

FIG. 1. (a) Scheme of the experiment. (b) Temporal sequence for the two atoms. Qualitative plot of the detuning $\Delta$ between the atomic transition frequency and mode $M_{a}$ versus time.

FIG. 2. Probability $P_{e}(T)$ for detecting $A_{p}$ in state $e$ as a function of the time interval $T$. Figures (a), (b), (c) and (d) correspond to four different time windows in the $0 \rightarrow 710 \mu$ s time interval. The dots are experimental, with errors bars reflecting the binomial statistics variance. The curves are sine fits. 